\title{
Anabases
}

ANABASES Traditions et réceptions de l'Antiquité

23 | 2016

Varia

\section{Luca ASMONTI, Athenian Democracy. A Sourcebook}

\author{
Claudine Leduc
}

(2) OpenEdition

Journals

Édition électronique

URL : http://journals.openedition.org/anabases/5680

DOI : 10.4000/anabases.5680

ISSN : 2256-9421

Éditeur

E.R.A.S.M.E.

Édition imprimée

Date de publication : 2 mai 2016

Pagination : 301-302

ISSN : 1774-4296

Référence électronique

Claudine Leduc, «Luca asmontı, Athenian Democracy. A Sourcebook », Anabases [En ligne], 23 | 2016, mis en ligne le 02 mai 2016, consulté le 23 septembre 2020. URL : http://journals.openedition.org/ anabases/5680; DOI : https://doi.org/10.4000/anabases.5680

Ce document a été généré automatiquement le 23 septembre 2020.

(c) Anabases 


\title{
Luca ASMONTI, Athenian Democracy. A Sourcebook
}

\author{
Claudine Leduc
}

\section{RÉFÉRENCE}

Luca ASMONTI, Athenian Democracy. A Sourcebook,

London-New Delhi-New York-Sydney, Bloomsbury sources in Ancient History, 2015,

$246 \mathrm{p}$.

120 dollars/ ISBN 978-0-8264-2034-3(hardback) ; 39, 95 dollars/ ISBN 978-161-4411-1371-9

(paperback) ; 29, 99 dollars/

ISBN 978-1-4411-6531-2 (eBook)

1 Luca Asmondi enseigne à l'Université du Queensland, en Australie. Ses recherches portent sur la démocratie athénienne au $\mathrm{V}^{\mathrm{e}}-\mathrm{IV}^{\mathrm{e}} \mathrm{s}$. av J.-C., sur les relations entre les Grecs et les Perses après la guerre du Péloponnèse et sur l'éloquence politique à Athènes. Comme il le dit lui-même (p. 1), cet ouvrage n'est qu'une anthologie destinée à initier à " la culture et à la pratique de la démocratie dans l'ancienne Athènes ». Il est conçu pour les étudiants qui abordent l'histoire ancienne classique et pour quiconque s'intéresse à l'histoire de la démocratie. Sans prétendre à l'exhaustivité, il croise, sur chaque question abordée, textes et inscriptions de base. Toutes les occurrences sélectionnées sont précédées d'une courte présentation et une bibliographie nourrie mais essentiellement en langue anglaise (sont exceptionnellement mentionnés quelques ouvrages traduits), accompagne les thèmes abordés.

2 L'introduction (12 pages) porte sur quatre points : les « messy realities » de la démocratie athénienne (elle ne correspond pas à nos critères; elle fut un régime contesté ; sa politeia est le résultat d'une série de conflits et de metabolai, de changements, comme dit [Aristote] dans la Constitution des Athéniens) ; le kratos du demos et l'énumération des principales institutions ; les limites de la démocratie ; la 
pertinence de l'étude de la démocratie athénienne : un système qui s'est toujours remis en question.

3 Pour tout enseignant chargé des débutants, la présentation de la démocratie athénienne est toujours très complexe. Comme le laisse présager, dès l'introduction, son intérêt pour les metabolai, l'auteur a décidé de construire son anthologie à partir des grands « changements » sur lesquels s'articule la première partie de la Constitution des Athéniens. Ce choix entraîne, me semble-t-il, deux difficultés : d'une part, le début de l'ouvrage a disparu, d'autre part [Aristote] est persuadé qu'une fois la démocratie restaurée en 403, il n'y a pas eu de " changement » au IV ${ }^{\mathrm{e}}$ siècle. Chapeauter la politeia primitive des archontes avec le "mythe " de Thésée risque de poser un délicat problème de méthode aux étudiants débutants qui vont devoir passer brutalement de l'analyse d'un récit fictif à celle d'un récit factuel. Est-il possible de faire de la démocratie $\mathrm{au} \mathrm{IV}^{\mathrm{e}}$ siècle un addenda de celle $\mathrm{du} \mathrm{v}^{\mathrm{e}}$ ? C'est précisément le moment, comme le montre la seconde partie de la Constitution des Athéniens, où les sources permettent de saisir comment, à travers le jeu des institutions, s'exerce, en démocratie, la « liberté » du citoyen. Pour ma part, il m'a toujours semblé difficile d'aborder la démocratie athénienne par l'analyse de la crise des $\mathrm{VII}^{\mathrm{e}}-\mathrm{VI}^{\mathrm{e}}$ siècle. Ne serait-ce pas avec l'analyse de cette crise - la liquidation du système palatial - qu'il est possible de mettre en évidence le contexte historique très spécifique d'un système politique qui n'est pas le nôtre?

L'auteur fait précéder les documents concernant les metabolai de la politeia d'un chapitre, à mon avis absolument essentiel, où il rassemble, en faisant bien sûr de larges emprunts à la Politique, les textes répondant aux trois questions : qu'est-ce que la polis ? Qu'est-ce que le citoyen ? Qu'est-ce que la liberté du citoyen ? Pour définir la polis, l'auteur ne retient que la définition proposée par Aristote au livre i de la Politique : la polis est la koinonia qui enveloppe toutes les autres koinoniai. Or, pour Aristote, polis est " un mot qui se dit en plusieurs sens » suivant la catégorie dans lequel il est analysé. Polis se dit donc aussi plethos politôn, la masse dénombrable des citoyens. Je me demande si ce n'est pas à partir de cette notion - une quantité qui se divise - qu'il faut aborder les définitions aristotéliciennes du citoyen et de sa " liberté ». Comme le maître le dit lui-même, celles qu'il propose ne sont valables qu'en démocratie.

Depuis les colloques qui ont commémoré les 2500 ans de la politeia de Clisthène qui fonda la démocratie, la question de la pertinence d'une référence à la démocratie athénienne est régulièrement posée. Pour l'auteur il s'agit d'un système imparfait mais qui a le mérite de s'être continuellement remis en question. Or il me semble que la démocratie athénienne est trop enracinée dans son contexte historique pour nous servir de modèle. Cela dit, les concepts qu'elle a élaborés sont toujours les nôtres et leur maniement demeure, si j'en crois mon expérience, extrêmement formateur pour des étudiants débutants ! 


\section{AUTEURS}

\section{CLAUDINE LEDUC}

Université Toulouse-Jean Jaurès (UT2J)

jobleduc@wanadoo.fr 